\title{
Concurrence-based entanglement measure for Werner States
}

\author{
Kai Chen ${ }^{1}$, Sergio Albeverio ${ }^{1}$, and Shao-Ming Fei ${ }^{1,2}$ \\ ${ }^{1}$ Institut für Angewandte Mathematik, Universität Bonn, D-53115, Germany \\ ${ }^{2}$ Department of Mathematics, Capital Normal University, Beijing 100037, China
}

\begin{abstract}
We give explicit expressions for entanglement measures of Werner states in arbitrary dimensions in terms of concurrence and tangle. We show that an optimal ensemble decomposition for a joint density matrix of a Werner state can achieve the minimum average concurrence and tangle simultaneously. Furthermore, the same decomposition also attains entanglement of formation for Werner states.
\end{abstract}

Keywords: Quantum information, Werner states, Entanglement measure, Concurrence, Tangle

Quantum entanglement is playing very significant roles in quantum information processing such as quantum cryptography, quantum teleportation and quantum computation [1]. This motivates an increasing interest in the study of operational detection and quantification of entanglement for various quantum systems. Despite of a great deal of efforts in recent years, for the moment only partial solutions are known to detect and quantify entanglement for generic mixed state.

The crucial entanglement measure concurrence, firstly proposed by Hill and Wootters [2, 3], has recently been shown to play an essential role in describing quantum phase transitions in various interacting quantum manybody systems [4], affecting macroscopic properties of solids significantly [5] and revealing distinct scaling behavior for different types of multipartite entanglement [6]. The concurrence was then generalized by Uhlmann, Rungta et al, and by Albeverio and Fei 7] to arbitrary bipartite quantum system. Multi-variable concurrence vectors are also introduced in [8, 9] and possible multipartite generalizations are given in [10].

However, even the problem of obtaining only lower bound of concurrence has required considerable efforts [8, 11]. This problem has been advanced significantly in [12], providing an algebraic lower bound which can be optimized further by numerical approaches, and in [13] through an entirely analytical derivation of a complementary tightly lower bound. In addition, nice analytical results are also given for isotropic states [14] and rotationally symmetric states [15].

An important class of quantum states are the Werner states [16, 17], which appear in realistic quantum computing devices and quantum communication environments, e.g. transmitting perfect entangled states through a noisy depolarizing channel. An effective experimental generation of these states has been recently demonstrated in [18]. An analytical expression has been derived in [17] for entanglement of formation (EOF), which quantifies the minimally required physical resources to prepare a Werner state. The greatest cross norm is also obtained for the Werner states [19]. It is believed [20] that there is a novel connection between the concurrence and their EOF, through a parameter that depicts the Werner state completely. One expects that the situation would be similar to the case of two qubits where EOF is an an- alytic monotone function of concurrence [3]. However, for Werner states why such a parameter plays the role of concurrence is not yet well understood. There is also no rigorous and clear proof of this fact in the literatures, for high dimensions.

In this letter we first find an analytic expression of concurrence for Werner states in arbitrary dimensions, which complements many of the existing analytic results. We then show how EOF is exactly related to the concurrence. We demonstrate that, surprisingly, an optimal ensemble decomposition will achieve concurrence, tangle and EOF simultaneously for the Werner states. Thus the expected connection is rigorously proved and shown to be natural.

Werner states. The Werner states are a class of mixed states for $d \times d$ systems (two qudits with $d \geq 2$ ) which are invariant under the transformations $U \otimes U$, for any unitary transformation $U[16,17]$. The density matrix of these states can be expressed as

$$
\rho_{f}=\frac{1}{d^{3}-d}(d-f) \mathbb{I}+(d f-1) \mathbb{F},
$$

where $\mathbb{F}$ is the flip operator (or swap operator) defined by $\mathbb{F}(\phi \otimes \psi)=\psi \otimes \phi$. In the computational basis $|i j\rangle$, $\mathbb{F}$ is of the form $\mathbb{F}=\sum_{i, j}^{d}|i j\rangle\langle j i|$. Here $f$ is a constant $f=\langle\mathbb{F}\rangle \equiv \operatorname{Tr}\left(\mathbb{F} \rho_{f}\right)$ satisfying $-1 \leq f \leq 1$. Werner states are separable if and only if $f \geq 0$, as shown in [16, 17].

It is evident that the Werner states are invariant under the action of the LOCC "twirling" superoperator $\mathcal{T}$

$$
\mathcal{T}\left(\rho_{f}\right)=\int d U U \otimes U \rho_{f} U^{\dagger} \otimes U^{\dagger}=\rho_{f} .
$$

where $d U$ refers to the standard Haar measure on the unitary matrix group. Consider an arbitrary initial pure $d \otimes d$ quantum state of the standard Schmidt form

$|\psi\rangle=\sum_{i j} \Phi_{i j}|i j\rangle=\sum_{i} \sqrt{\mu_{i}}\left|a_{i} b_{i}\right\rangle=\left(U_{A} \otimes U_{B}\right) \sum_{i} \sqrt{\mu_{i}}|i i\rangle$,

where $\left|a_{i}\right\rangle$ and $\left|b_{i}\right\rangle$ are orthonormal bases of the subsystems $\mathcal{H}_{A}$ and $\mathcal{H}_{B}$, respectively. The state $|\psi\rangle$ is thus specified by its Schmidt vector $\vec{\mu} \equiv\left(\mu_{1}, \mu_{2}, \ldots, \mu_{d}\right)$ and the unitary operators $U_{A}$ and $U_{B}$. For convenience of later use, we use the symbol $\Phi$ to denote the pure state 
$|\psi\rangle$, where $\Phi$ is the matrix with entries $\Phi_{i j}$, which contains all the information for $|\psi\rangle$. In fact, any two-qudit $\rho$ reduces to a Werner state

$$
\mathcal{T}(\rho)=\rho_{f(\rho)},
$$

under the twirling superoperator while keeping $f(\rho)=$ $\langle\mathbb{F}\rangle=\operatorname{Tr}(\mathbb{F} \rho)$ invariant. This can easily be seen from

$$
\operatorname{Tr}(\mathbb{F} \mathcal{T}(\rho))=\operatorname{Tr}(\mathcal{T}(\mathbb{F}) \rho)=\operatorname{Tr}(\mathbb{F} \rho) .
$$

As for the pure state Eq. (3), one has

$$
\mathcal{T}(|\psi\rangle\langle\psi|)=\rho_{f}
$$

with $f$ given by

$$
f=\operatorname{Tr}(|\psi\rangle\langle\psi| \mathbb{F})=\sum_{i j} \Phi_{i j} \Phi_{j i}^{*} .
$$

Entanglement measures in terms of concurrence and tangle. The (generalized) definition [7] of concurrence $C(|\psi\rangle)$ for a pure state $|\psi\rangle$ is as follows: $C(|\psi\rangle)=\sqrt{2\left(1-\operatorname{Tr} \rho_{A}^{2}\right)}$, where the reduced density matrix $\rho_{A}$ is given by $\rho^{A}=\operatorname{tr}_{B}(|\psi\rangle\langle\psi|)$. This can then be extended to mixed states by the "convex roof construction",

$$
C(\rho) \equiv \min _{\left\{p_{i},\left|\psi_{i}\right\rangle\right\}} \sum_{i} p_{i} C\left(\left|\psi_{i}\right\rangle\right),
$$

where $\rho=\sum_{i} p_{i}\left|\psi_{i}\right\rangle\left\langle\psi_{i}\right|, p_{i} \geq 0$ and $\sum_{i} p_{i}=1$. For any pure product state $|\psi\rangle, C(|\psi\rangle)$ vanishes according to the definition. Consequently, a state $\rho$ is separable if and only if $C(\rho)=0$. A separable state can then be represented as a convex combination of product states [16].

Another entanglement measure called tangle, was first proposed in [21]. Its generalization to generic mixed states and further properties were explored in [14, 22]. The tangle $\tau(\rho)$ is by definition the squared concurrence for pure states, and can be similarly extended to mixed states

$$
\tau(\rho) \equiv \min _{\left\{p_{i},\left|\psi_{i}\right\rangle\right\}} \sum_{i} p_{i} C^{2}\left(\left|\psi_{i}\right\rangle\right)
$$

where $C^{2}\left(\left|\psi_{i}\right\rangle\right)$ stands for $\left(C\left(\left|\psi_{i}\right\rangle\right)\right)^{2}$.

For the pure state $|\psi\rangle$ of (3), we have:

$$
\begin{aligned}
\tau(\Phi) & =C^{2}(\Phi)=2\left(1-\sum_{i} \mu_{i}^{2}\right) \\
& =4 \sum_{i<j} \mu_{i} \mu_{j}=C^{2}(\vec{\mu})=\tau(\vec{\mu}),
\end{aligned}
$$

which varies smoothly from 0 , for pure product states, to $2(d-1) / d$ for maximally entangled pure states.

Concurrence and tangle for Werner states. To derive the tangle and concurrence for Werner states, we will use a technique developed in [14, 17, 23]. The EOF is defined to be $E(\rho) \equiv \min _{\left\{p_{i},\left|\psi_{i}\right\rangle\right\}} \sum_{i} p_{i} E\left(\left|\psi_{i}\right\rangle\right)$ for all possible ensemble realizations $\rho=\sum_{i} p_{i}\left|\psi_{i}\right\rangle\left\langle\psi_{i}\right|$, where $p_{i} \geq 0$ and $\sum_{i} p_{i}=1$. Here $E(|\psi\rangle)=S\left(\rho_{A}\right)$ with $S\left(\rho_{A}\right)$ the entropy $S\left(\rho_{A}\right) \equiv-\sum_{i=1}^{d} \mu_{i} \log _{2} \mu_{i}=H(\vec{\mu})$, where $\mu_{i}$ are all the eigenvalues of $\rho_{A}$ and $\vec{\mu}$ is the Schmidt vector $\left(\mu_{1}, \mu_{2}, \ldots, \mu_{d}\right)$. The EOF of Werner states is derived in 17] as being given by

$$
E\left(\rho_{f}\right)=H_{2}\left(\frac{1}{2}\left(1-\sqrt{1-f^{2}}\right)\right),
$$

by an elegant extremization procedure. Here $H_{2}($.$) is$ the binary entropy function. Since $E\left(\rho_{f}\right)$ is a monotonically increasing function of $-f$, as seen from Eq. (11), it is expected [20] that $-f$ plays the role of concurrence, similarly as in the two qubits case [3].

Simplification through symmetry. Before getting through possible extremization procedures, we first recall some formulations of the convex roof construction of entanglement measures 17, 24]. We denote by $K$ the whole set of states in a given quantum system and by $M$ the set of all pure states in $K$. Then the elements of $\mathrm{K}$ are convex linear combinations of a finite number elements of $M$. Let $G$ be a compact group of symmetries acting on $K$ by transformations $\alpha_{U}: \rho \mapsto U \rho U^{\dagger}$, $U$ being an element of $G$, and assume that a pure-state measure $E$ defined on $M$ is invariant under $G$. We define a projection $\mathbf{P}: K \rightarrow K$ by $\mathbf{P} \rho=\int d U U \rho U^{\dagger}$ with $d U$, as before, the standard Haar measure on $G$, and a function $\varepsilon$ on $\mathbf{P} K$ by

$$
\varepsilon(\rho)=\min \{E(|\psi\rangle):|\psi\rangle \in M, \mathbf{P}|\psi\rangle\langle\psi|=\rho\} .
$$

For $\rho \in \mathbf{P} K$, it is proved that [17]

$$
\operatorname{co} E(\rho)=\operatorname{co} \varepsilon(\rho),
$$

where cof at the right hand side stands for the convex hull construction for a given function $f$ restricted to the pure states satisfying $\mathbf{P}|\psi\rangle\langle\psi|=\rho$, as shown in Eq. (12). When we take the concurrence as the entanglement measure, $\operatorname{co} E(\rho)$ at the left hand side of Eq. (13) corresponds to

$$
\begin{aligned}
C(\rho) & =\operatorname{coC}(\rho) \\
& \left.=\min \left\{\sum_{i} p_{i} C\left(\left|\psi_{i}\right\rangle\right)\left|\rho=\sum_{i} p_{i}\right| \psi_{i}\right\rangle\left\langle\psi_{i}\right|\right\},
\end{aligned}
$$

where the infimum is taken over all possible convex combinations with $p_{i} \geq 0$ and $\sum_{i} p_{i}=1$.

According to the above results, in order to derive concurrence or tangle for the Werner states one thus needs only to consider all the pure states $\sigma$ satisfying $P \sigma=\rho$ and achieving minimal admissible concurrence or tangle for $\sigma$. Finally one computes their convex hull. Here and later by "minimal admissible", we mean the minimal value of concurrence or tangle among all possible ensemble decompositions of the density matrix. 
Extremization for pure states. With a given $f$ and the corresponding Werner state $\rho_{f}$, we are going to find the desired pure states $\sigma$ with coefficient matrix $\Phi$ satisfying $P \sigma=\rho_{f}$ and minimize $C^{2}(\Phi)$. The task amounts to the following problem:

$$
\begin{cases}\operatorname{minimize} & C^{2}(\Phi) \\ \text { subject to } & \sum_{i j} \Phi_{i j} \Phi_{j i}^{*}=f \\ & \sum_{i j}\left|\Phi_{i j}\right|^{2}=1\end{cases}
$$

The key point of our idea is to apply the concavity properties of both $\tau(\Phi)$ and $C(\Phi)$ with respect to the reduced density matrix $\rho^{A}=\operatorname{tr}_{B}(|\psi\rangle\langle\psi|)$, as proved in [14], i.e.

$$
g\left(\lambda_{1} \Phi_{1}+\lambda_{2} \Phi_{2}\right) \geq \lambda_{1} g\left(\Phi_{1}\right)+\lambda_{2} g\left(\Phi_{2}\right),
$$

where $\lambda_{1}, \lambda_{2} \geq 0, \lambda_{1}+\lambda_{2}=1$ and where $g$ can be $\tau(\Phi)$ as well as $C(\Phi)$. By using this property, we will derive tight lower bounds for $\tau(\Phi)$ resp. $C(\Phi)$, and then find a condition under which the bound is achieved. Thus an essential step for the minimization problem Eq. (15) is to find such a condition under which the tight lower bound is achieved.

From Eq. (3), one has the reduced density matrix $\rho^{A}=$ $\Phi \Phi^{\dagger}$. The $U \otimes U$ transformations will neither change the degree of entanglement of a state nor the constraint condition $\sum_{i j} \Phi_{i j} \Phi_{j i}^{*}=f$. In fact, it corresponds to a local unitary transformation in $\rho^{A}$, i.e., $\rho^{A} \longrightarrow U \rho^{A} U^{\dagger}$. Thus one can choose conveniently $\Phi$ such as to make $\rho^{A}$ diagonal. The eigenvalues of $\rho^{A}$ are then $\mu_{i}=\rho_{i i}^{A}=$ $\sum_{k}\left|\Phi_{i k}\right|^{2}$. From Eq. (10), the tangle is of the form

$$
\tau(\Phi)=2\left(1-\sum_{i}\left(\sum_{k}\left|\Phi_{i k}\right|^{2}\right)^{2}\right)
$$

It is helpful to look at the eigenvalues of $\rho^{A}$ as a distribution of $d$ random variables

$$
S=\left(\sum_{k}\left|\Phi_{1 k}\right|^{2}, \sum_{k}\left|\Phi_{2 k}\right|^{2}, \ldots, \sum_{k}\left|\Phi_{d k}\right|^{2}\right),
$$

which is a convex combination of the distributions

$$
\begin{aligned}
& S_{i j}=(\underbrace{0, \ldots, 0}_{i-1},\left|\Phi_{i j}\right|^{2}, \underbrace{0, \ldots, 0}_{j-i-1},\left|\Phi_{j i}\right|^{2}, \underbrace{0, \ldots, 0}_{d-j}) / p_{i j}, \\
& \text { with probability } p_{i j}=\left|\Phi_{i j}\right|^{2}+\left|\Phi_{j i}\right|^{2} \text {, } \\
& S_{i i}=(\underbrace{0, \ldots, 0}_{i-1}, 1, \underbrace{0, \ldots, 0}_{d-i}) \text {, } \\
& \text { with probability } p_{i i}=\left|\Phi_{i i}\right|^{2} \text {, }
\end{aligned}
$$

where $\sum_{i \leq j} p_{i j}=1$ and $i<j \leq d$. Hence

$$
S=\sum_{i \leq j} p_{i j} S_{i j}
$$

Exploiting the concavity property of $\tau(\Phi)$, we get

$$
\tau(S) \geq \sum_{i \leq j} p_{i j} \tau\left(S_{i j}\right)=\sum_{i<j} p_{i j} \tau\left(S_{i j}\right),
$$

where we have used $\tau\left(S_{i i}\right)=0$. On the other hand, the function $f$ in Eq. (7) can be similarly expressed as

$$
f=\sum_{i \leq j} p_{i j} f_{i j}
$$

where

$$
\begin{array}{rlr}
f_{i j} & =\left(\Phi_{i j} \Phi_{j i}^{*}+\Phi_{j i} \Phi_{i j}^{*}\right) / p_{i j} \\
& =2 \operatorname{Re}\left(\Phi_{i j} \Phi_{j i}^{*}\right) / p_{i j}, \quad \text { for } i<j \\
f_{i i} & =1 .
\end{array}
$$

We now look for a lower bound of $\tau\left(S_{i j}\right)$ for a given $f_{i j}$. Set $x=\Phi_{i j} / \sqrt{p_{i j}}, y=\Phi_{j i} / \sqrt{p_{i j}}$, Minimizing $\tau\left(S_{i j}\right)$ is equivalent to

$$
\begin{cases}\operatorname{minimize} & \tau\left(S_{i j}\right)=4|x y|^{2}=4|x|^{2}\left(1-|x|^{2}\right) \\ \text { subject to } & 2 \operatorname{Re}\left(x y^{*}\right)=f_{i j} \\ & |x|^{2}+|y|^{2}=1\end{cases}
$$

Since $4|x|^{2}\left(1-|x|^{2}\right)$ is a monotonically increasing function of $|x|^{2}$ taking values from 0 to $1 / 2$, minimizing $\tau\left(S_{i j}\right)$ is equivalent to minimizing $|x|^{2}$ for given $f_{i j}$. This kind of problem was solved in [17], and the solution is $|x|_{\min }^{2}=$ $\left(1-\sqrt{1-f_{i j}^{2}}\right) / 2$. Thus $\tau\left(S_{i j}\right) \geq 4|x|_{\min }^{2}\left(1-|x|_{\min }^{2}\right)=f_{i j}^{2}$. From Eq. (22), one has further

$$
\begin{aligned}
\tau(S) & \geq \sum_{i<j} p_{i j} \tau\left(S_{i j}\right) \geq \sum_{i<j} p_{i j} f_{i j}^{2} \geq\left(\sum_{i<j} p_{i j} f_{i j}\right)^{2} \\
& =\left(f-\sum_{i} p_{i i}\right)^{2}=\left(f-\sum_{i}\left|\Phi_{i i}\right|^{2}\right)^{2}
\end{aligned}
$$

where we have used the convexity property of $f_{i j}^{2}$ in the third inequality of Eq. (27).

Case 1: $f \geq 0$

$\tau(S)$ itself vanishes if there is only one nonzero eigenvalue 1 of $\rho^{A}$, say

$$
\rho_{i i}^{A}=\sum_{k}\left|\Phi_{i k}\right|^{2}=1
$$

The minimum $\left(f-\sum_{i}\left|\Phi_{i i}\right|^{2}\right)^{2}$ will be 0 if one chooses in addition

$$
\left|\Phi_{i i}\right|^{2}=f
$$

The two equations Eqs. (28) and (29) can always be satisfied by a suitable choice of $\Phi$. Thus the minimal admissible value for $\tau(S)$ is 0 .

Case 2: $f<0$

It is clear that any choice of nonzero $\Phi_{i i}$ will increase the value of $\left(f-\sum_{i}\left|\Phi_{i i}\right|^{2}\right)^{2}$. Therefore for an optimal solution one should have all $\Phi_{i i}=0$, if possible. On the other hand, the equalities in Eq. (27) hold, if there is one single item in the summation, due to the concavity property of $\tau(S)$ (the first inequality in Eq. (27)) and 
the convexity of $f^{2}$ (the third inequality in Eq. (27)). This is because all the inequalities will become equalities $\tau(S)=\tau\left(S_{i j}\right)=f_{i j}^{2}=f^{2}$ when $p_{i j}=1$. Therefore we have two nonzero components left, say $\Phi_{i j}$ and $\Phi_{j i}$. Hence one has

$$
\begin{aligned}
\Phi_{i j} \Phi_{j i}^{*}+\Phi_{j i} \Phi_{i j}^{*} & =2 \operatorname{Re}\left(\Phi_{i j} \Phi_{j i}^{*}\right)=f \\
\left|\Phi_{i j}\right|^{2}+\left|\Phi_{j i}\right|^{2} & =1
\end{aligned}
$$

and thus

$$
\begin{aligned}
& \Phi_{i j}=e^{i \theta_{1}}\left(\left(1-\sqrt{1-f^{2}}\right) / 2\right)^{\frac{1}{2}} \\
& \Phi_{j i}=e^{i \theta_{2}}\left(\left(1+\sqrt{1-f^{2}}\right) / 2\right)^{\frac{1}{2}}
\end{aligned}
$$

where $\theta_{1,2}$ are arbitrary real numbers satisfying $\theta_{1}-\theta_{2}=$ $(2 n+1) \pi$, with $n$ being any integer. With these choices of $\Phi_{i j}$ and $\Phi_{j i}$ in Eq. (30), one gets the minimal admissible value of $\tau_{\min }(S)=f^{2}$.

Combining all the above results, we have

$$
\tau_{\min }(\Phi)= \begin{cases}f^{2}, & \text { for } f<0 \\ 0, & \text { for } f \geq 0\end{cases}
$$

for $\Phi$ satisfying $P|\Phi\rangle\langle\Phi|=\rho_{f}$.

Since $C(\Phi)$ is also concave and is a monotonously increasing function of $\tau(\Phi)$, we have similar expressions as in Eqs. (22) and (27),

$$
\begin{aligned}
C(S) & \geq \sum_{i \leq j} p_{i j} C\left(S_{i j}\right)=\sum_{i<j} p_{i j} C\left(S_{i j}\right) \\
& \geq \sum_{i<j} p_{i j}\left|f_{i j}\right| \geq\left|\sum_{i<j} p_{i j} f_{i j}\right| \\
& =\left|f-\sum_{i} p_{i i}\right|=\left.\left|f-\sum_{i}\right| \Phi_{i i}\right|^{2} \mid .
\end{aligned}
$$

The above analysis for minimizing tangle $\tau(S)$ can naturally be extended to the minimization of $C(S)$. It is evident that the solution of Eq. (26) also achieves the minimal admissible value

$$
C_{\min }(\Phi)= \begin{cases}|f|=-f, & \text { for } f<0 \\ 0, & \text { for } f \geq 0\end{cases}
$$

for $\Phi$ satisfying $P|\Phi\rangle\langle\Phi|=\rho_{f}$.

Remark: It is shown in [25] that different entanglement measures will produce the same ordering for pure states if they reduce to the entropy of entanglement for pure states. However, the concurrence and tangle do not belong to that class. In fact, they will generally lead to different orderings when compared with EOF for pure states, since there are no simple monotonous function relations among them and the $\operatorname{EOF} E(\Phi)$ (except for an apparent connection $E(\Phi)=H_{2}\left(\frac{1}{2}\left(1-\sqrt{1-C(\Phi)^{2}}\right)\right)$ holding only for $2 \otimes N$ systems as easily seen from the definition). This means that a state $\Phi$ achieving a minimal $E(\Phi)$ may not automatically produce a minimal $\tau(\Phi)$ or $C(\Phi)$. In our case of Werner states, it occurs by chance that the solution Eq. (30) achieves minima for all of the three entanglement measures.

With the above derived results, we can now calculate the concurrence and tangle. This is the content of the following Theorem.

Theorem: The concurrence $C\left(\rho_{f}\right)$ resp. tangle $\tau\left(\rho_{f}\right)$ for the Werner states $\rho_{f}$ of Eq. (1) are given by

$$
\left\{\begin{array}{l}
C\left(\rho_{f}\right)=-f, \\
r e s p . \\
\tau\left(\rho_{f}\right)=f^{2} .
\end{array}\right.
$$

for $f<0$ and $C\left(\rho_{f}\right)=\tau\left(\rho_{f}\right)=0$ for $f \geq 0$.

Proof: It is evident that both concurrence and tangle will be 0 according to the convex hull construction of Eqs. (31) and (33) for $f \geq 0$. We focus on the case where $-1 \leq f<0$, which implies that the Werner states are entangled. For any pure state $\sigma$ of Eq. (3) satisfying $P \sigma=\rho_{f}$, we have already found that the minimal admissible values for $\tau(\sigma)$ and $C(\sigma)$ are given by Eqs. (31) and (33). The optimal choice for $\sigma=|\Phi\rangle\langle\Phi|$ is given by Eq. (30).

Now we can compute the convex hull of the function $C(\sigma)$ (or $\left.\tau(\sigma)=C^{2}(\sigma)\right)$ through the results of Eqs. (12), (13) and (14)). We have:

$$
\begin{cases}\frac{\partial C(\sigma)}{\partial f}=-1<0, & \frac{\partial^{2} C(\sigma)}{\partial f^{2}}=0, \\ \frac{\partial C^{2}(\sigma)}{\partial f}=2 f<0, & \frac{\partial^{2} C^{2}(\sigma)}{\partial f^{2}}=2>0 .\end{cases}
$$

Thus both $C(\sigma)$ and $C^{2}(\sigma)$ are monotonically convex functions of $f$. For the Werner states $\rho_{f}$, which is a convex combination of the states $\sigma$, one has naturally the results of Eqs. (34) according to the convex hull construction.

It is shown in [17] that any pure state in the optimal decomposition that achieves EOF has the form of Eq. (30). The solution can also be rephrased to have Schmidt rank 2 and Schmidt coefficients $\mu_{1}=\left(1+\sqrt{1-f^{2}}\right) / 2$ and $\mu_{2}=\left(1-\sqrt{1-f^{2}}\right) / 2$. Thus the optimal decomposition for achieving concurrence and tangle also achieves EOF at the same time. This shows that all of the three entanglement measures share a common important feature, namely to give the same values for every pure state in the optimal ensemble decomposition. In addition, the relation shown in Eq. (11) that EOF is a monotonically increasing function of the concurrence holds naturally, since every pure state in the optimal ensemble decomposition has the same Schmidt number 2. This is similar to the two qubits case [2, 3] where every pure state in an optimal ensemble decomposition does have the same value of concurrence or EOF. Our results thus give the first rigorous proof for the common "belief" that, for Werner states, $-f$ plays exactly the role of concurrence.

In summary, we have given an entirely analytic derivation of the concurrence and tangle for the Werner states. 
Our results show that the concurrence, tangle and entanglement of formation have the same optimal decomposition. This is very different from the isotropic case [14, 23], where the tangle and EOF have a similar behavior while the concurrence behaves in a completely different manner. This implies that the Werner states have a more subtle entanglement structure than the isotropic states, though basically they are partial transpositions of each other, with respect to one subsystem in some parameter ranges [17]. Since concurrence is a good entanglement measure and can reveal many important physical features of the systems involved, our results would shed new light on a deeper understanding of entanglement.

Acknowledgments. K.C. gratefully acknowledges support from the Alexander von Humboldt Foundation. This work has been supported the Deutsche Forschungsgemeinschaft SFB611 and German(DFG)Chinese(NSFC) Exchange Program 446CHV113/231. K.C. also thanks hospitality of Department of Mathematics in Capital Normal University where part of this work was finished.
[1] M.A. Nielsen and I.L. Chuang, Quantum Computation and Quantum Information, Cambridge University Press, Cambridge, 2000.

[2] S. Hill and W.K. Wootters, Phys. Rev. Lett. 78, 5022 (1998).

[3] W.K. Wootters, Phys. Rev. Lett. 80, 2245 (1998).

[4] A. Osterloh, L. Amico, G. Falci and R. Fazio, Nature 416, 608 (2002); L.-A. Wu, M.S. Sarandy, and D.A. Lidar, Phys. Rev. Lett. 93, 250404 (2004).

[5] S. Ghosh, T.F. Rosenbaum, G. Aeppli, S.N. Coppersmith, Nature 425, 48 (2003); V. Vedral, Nature 425, 28 (2003).

[6] A.R.R. Carvalho, F. Mintert, and A. Buchleitner, Phys. Rev. Lett. 93, 230501 (2004).

[7] A. Uhlmann, Phys. Rev. A 62, 032307 (2000); P. Rungta, V. Buzek, C.M. Caves, M. Hillery, and G.J. Milburn, Phys. Rev. A 64, 042315 (2001); S. Albeverio and S. M. Fei, J. Opt. B: Quantum Semiclassical Opt. 3, 223 (2001).

[8] K. Audenaert, F. Verstraete, and B. De Moor, Phys. Rev. A 64, 052304 (2001).

[9] P. Badziag, P. Deuar, M. Horodecki, P. Horodecki, and R. Horodecki, J. Mod. Opt. 49, 1289 (2002); H. Fan, K. Matsumoto, and H. Imai, J. Phys. A: Math. Gen. 36, 4151 (2003); G. Gour, Phys. Rev. A 71, 012318 (2005).

[10] S.J. Akhtarshenas, J. Phys. A: Math. Gen. 38, 6777 (2005); F. Mintert, M. Kuś, and A. Buchleitner, Phys. Rev. Lett. 95, 260502 (2005).

[11] P.X. Chen, L.M. Liang, C.Z. Li and M.Q. Huang, Phys. Lett. A 295175 (2002); E. Gerjuoy, Phys. Rev. A 67052308 (2003); A. Łoziński, A. Buchleitner, K. Zy- czkowski, and T. Wellens, Europhys. Lett. 62168 (2003).

[12] F. Mintert, M. Kuś, and A. Buchleitner, Phys. Rev. Lett. 92, 167902 (2004); F. Mintert, Ph.D. thesis, Measures and dynamics of entangled states, Munich University, Munich, 2004; F. Mintert, A.R.R. Carvalho, M. Kuś, and A. Buchleitner, Phys. Rep. 415, 207 (2005).

[13] K. Chen, S. Albeverio, and S.M. Fei, Phys. Rev. Lett. 95, 040504 (2005).

[14] P. Rungta and C.M. Caves, Phys. Rev. A 67, 012307 (2003).

[15] K.K. Manne and C.M. Caves, e-print: quant-ph/0506151.

[16] R.F. Werner, Phys. Rev. A 40, 4277 (1989).

[17] K.G.H. Vollbrecht and R.F. Werner, Phys. Rev. A 64, 062307 (2001).

[18] Y.S. Zhang, Y.F. Huang, C.F. Li, and G.C. Guo, Phys. Rev. A 66, 062315 (2002).

[19] O. Rudolph, quant-ph/0202121.

[20] W.K. Wootters, Quant. Inf. Comp. 1, 27 (2001).

[21] V. Coffman, J. Kundu, and W.K. Wootters, Phys. Rev. A 61, 052306 (2000).

[22] T.J. Osborne, Phys. Rev. A 72, 022309 (2005).

[23] B.M. Terhal and K.G.H. Vollbrecht, Phys. Rev. Lett. 85, 2625 (2000).

[24] S. Lee, D.P. Chi, S.D. Oh, and J. Kim, Phys. Rev. A. 68, 062304 (2003).

[25] S. Virmani and M.B. Plenio, Phys. Lett. A 268, 31 (2000). 